\title{
Nutritional risk assessment and cultural validation of the modified NUTRIC score in critically ill patients-A multicenter prospective cohort study
}

\author{
Rosa Mendes ${ }^{\mathrm{a}, *}$, Sara Policarpo ${ }^{\mathrm{b}}$, Philip Fortuna ${ }^{\mathrm{a}}$, Marta Alves ${ }^{\mathrm{c}}$, \\ Daniel Virella ${ }^{c}$, Daren K. Heyland ${ }^{\mathrm{d}}$ the Portuguese NUTRIC Study Group \\ a Medical Urgency Unit (General Intensive Care Unit), Hospital S. José, Centro Hospitalar de Lisboa Central, Lisbon, Portugal \\ b Dietetics and Nutrition Department, Hospital Universitário de Santa Maria, Centro Hospitalar de Lisboa Norte, Lisbon, Portugal \\ ' Research Unit, Centro Hospitalar de Lisboa Central, Lisbon, Portugal \\ d Clinical Evaluation Research Unit, Kingston General Hospital, Kingston, Ontario, Canada
}

\section{A R T I C L E I N F O}

Keywords:

Critically ill

Nutritional risk

Modified NUTRIC score

Effectiveness

Cultural validation

\begin{abstract}
A B S T R A C T
Purpose: Characterize the nutritional risk of critically ill patients with the modified NUTrition Risk in the Critically ill (NUTRIC) score.

Materials: National, multicenter, prospective, observational study conducted in 15 polyvalent Portuguese intensive care unit (ICU), during 6 months. Adult patients were eligible. Those transferred from another ICU or readmitted, brain dead at admission, and with length of ICU stay (LOS) of 72 hours or less were excluded. NUTRIC score was calculated at admission; scores $\geq 5$ represent a high nutritional risk. Main outcome was mortality from all causes at 28 days after admission to the ICU; LOS and days without mechanical ventilation (days free of MV) were secondary outcomes.

Results: From 2061 admissions, 1143 patients were considered, mostly males ( $\mathrm{n}=744,64.7 \%$ ) with median $\left(\mathrm{P}_{25}-\mathrm{P}_{75}\right)$ age of 64 (51-75). Patients at high nutritional risk were 555 (48.6\%). High NUTRIC score was associated with longer $\operatorname{LOS}(P<.001)$, less days free of MV $(P=.002)$ and higher 28-day mortality $(P<.001)$. The area under the curve of NUTRIC score $\geq 5$ for predicting 28 -day mortality was 0.658 ( $95 \% \mathrm{CI}, 0.620-0.696$ ). NUTRIC score $\geq 5$ had a positive predictive value $32.7 \%$ and a negative predictive value $88.8 \%$ for 28 -day mortality. Conclusions: Almost half of the patients in Portuguese ICUs are at high nutritional risk. NUTRIC score was strongly associated with main clinical outcomes.
\end{abstract}

(c) 2016 Elsevier Inc. All rights reserved.

\section{Introduction}

Malnutrition is common in hospitalized patients and highly prevalent in the population of critically ill patients all around the world $[1,2]$. It is associated with increased morbidity, mortality and occurrence of nosocomial infections, prolonged hospitalization, worse functional status at discharge from the Intensive Care Units (ICU) and increased hospital costs $[3,4]$.

In Portugal, data regarding the impact of malnutrition at hospital admission has demonstrated that the frequency of patients at nutritional risk is very high, comprising $29 \%$ to $47 \%$, depending on the methodology used [5]. Data on Portuguese critically ill patients is still, to date, not available.

Most of the scores and tools to assess nutritional risk were validated in the hospital setting [6-12], and include a variety of criteria to identify

\footnotetext{
* Corresponding author at: Unidade de Urgência Médica, Hospital S. José, CHLC, Rua José Serrano, 1150-199 Lisboa, Portugal. Tel.: + 351218841303.

E-mail address: rosamariamendes@gmail.com (R. Mendes).
}

nutritional risk, such as food/nutritional intake, physical examination, severity of illness, anthropometric data and functional assessment. Many of these criteria are difficult to obtain in critically ill patients since almost all of these patients require mechanical ventilation and sedation. Changes in weight can be influenced by fluid status, given the large volumes necessary to maintain hemodynamic stability, and consequently muscle and fat wasting evaluation become more difficult. Many traditional tools do not provide information regarding inflammatory status which is crucial in an ICU population, since it's one of the factors responsible for hypermetabolic status and hence, muscle wasting [13].

Based on the assumption that the nutritional risk is not the same for all critically ill patients, Heyland et al developed and validated the NUTrition Risk in the Critically ill (NUTRIC score), the first nutritional risk assessment tool developed specifically for the ICU population that could identify patients that require more aggressive nutritional support, based on their nutritional risk $[14,15]$. The conceptual model links patient predictor markers of acute and chronic starvation, acute and chronic inflammation and outcome. The severity of illness is derived from the use of the variables with traditional scores of severity of illness, 
the Acute Physiology and Chronic Health Evaluation II (APACHE II) score [16] and baseline Sequential Organ Failure Assessment (SOFA) [17].

The variables in the NUTRIC score are easy to obtain in the critical care setting, except Interleukin-6 (IL-6) level, which is not commonly measured. As it has already been demonstrated, the performance of the NUTRIC score varies only slightly when excluding IL-6 levels from the score or when this is replaced by another available inflammatory biomarker. The modified NUTRIC score (without IL-6) has been recently validated [18].

\section{Objective}

To characterize the nutritional risk of hospitalized patients in Portuguese ICU using the modified NUTRIC score.

\section{Materials}

A prospective, observational, multicentric cohort study was conducted in a convenient sample of tertiary polyvalent ICUs across Portugal. Nationwide, around 4100 patients per year were expected to be admitted in the ICUs. Polyvalent ICUs from public academic hospitals were considered eligible to participate. From the 30 identified ICUs, 23 expressed the will to participate and 15 ICUs provided the complete dataset for effective enrolment. All enrolled ICUs had allocated nutritional staff. Patients consecutively admitted to the participant ICUs were enrolled during a period of 6 months, in 2014, with only admission to the ICU being considered. Patients aged over 18 years were considered eligible for recruitment. Patients were excluded if diagnosed as being brain dead at admission or if they had been transferred from another ICU or readmitted into the ICU. Only patients with a length of ICU stay (LOS) of more than 72 hours were considered for analysis.

The transcultural adaptation of the tool was previously done as an independent part of the project. The process of cross-cultural adaptation followed the multistep approach, according to the international guidelines [19]. The original English version of the NUTRIC score was independently translated to Portuguese by two bilingual translators with specific skills in English and Portuguese and proved experience in the health sciences. A panel of experts (physicians, nurses and dietitians working in ICU) evaluated the two translations of NUTRIC score, by analyzing the phrasing of each item, and consensually obtaining the proposed version. An official translator, registered in the Portuguese Translators Society, proceeded to back-translate the proposed version to English, the language of the original version. The back-translated version was reviewed by the developers of the original tool to assess the adequateness of the content (content validity). A pilot study was conducted with 46 critically ill patients admitted in one of the ICU's from the study, to assess the understanding and applicability of the translated version of the modified NUTRIC Score. The cultural validation from English to Portuguese language has succeeded in achieving idiomatic, semantic, and conceptual equivalence between the original toll and the Portuguese version. The official endorsement of the proposed Portuguese version of the NUTRIC score was obtained.

The NUTRIC score, without IL-6 levels includes five variables: age, APACHE II, SOFA, number of co-morbidities and days from hospital to ICU admission. The score was calculated with data from the first $24 \mathrm{~h}$ after ICU admission. The NUTRC score ranges from zero to nine; a score $\geq 5$ indicates a high nutritional risk [14]. Main outcome was mortality from all causes at 28 days after admission to the ICU; LOS and days without invasive mechanical ventilation (days free of MV) were secondary outcomes. Outcomes were collected until day 28, starting at admission to the ICU. Data were recorded from the patient chart (electronic and/or paper) following the standardized data collection procedures stated in the manual of procedures developed specifically for the study. Local researchers received training to collect data. The study was approved by the Ethics Committees for Health for every participant Hospitals and was licensed in the Portuguese Data Protection Authority (no. 6635/2013). As mandated by the same Authority, informed consent was obtained from all patients included.

Prevalence rates are presented with 95\% confidence intervals (95\% CI). Proportions are compared with binomial test, $\chi^{2}$ test or Fisher exact test (as adequate). The normality of the distribution of the NUTRIC score was excluded. The association of the NUTRIC score with the three main outcomes (LOS, days off MV and 28-day mortality) was analyzed with Mann-Whitney $U$ test and Spearman correlation, adjusted to confounding factors when needed. Logistic regression analysis was used to further characterize the association between the NUTRIC score and the three main outcomes, using odds ratio (OR) with $95 \% \mathrm{CI}$ of the estimates; linear regression was performed but discarded due to rejection of the normality of the residuals. The model discrimination for predicting 28-day mortality was assessed by the area under the receiving operating characteristic curve (AUC) (interpretation: excellent $\geq 0.90$, adequate $0.70-0.89$, poor $<0.70$ ) and the generalized maxrescaled R-squared statistic.

The following software packages were used for analysis: OpenEpi (Dean AG, Sullivan KM, Soe MM. OpenEpi: Open Source Epidemiologic Statistics for Public Health. www.OpenEpi.com, Version 2015/05/04, accessed 2015/07/25), SISA (Uitenbroek, Daan G. SISA. 1997. http:// www.quantitativeskills.com/sisa.htm. (2015/07/25)), SPSS 22.0 (SPSS for Windows, Rel. 22.0.1. 2013. SPSS Inc, Chicago, IL; EUA), Stata (StataCorp. 2013. Stata Statistical Software: Release 13. College Station, TX; StataCorp LP, USA) and VassarStats (Lowry R. VassarStats. http:// vassarstats.net/. Vassar College, Poughkeepsie, NY, USA, accessed 2015/07/25).

\section{Results}

During the 6-month recruitment period, 2061 patients were eligible for enrolment in the 15 participating ICUs and 1143 were included in the analysis. Exclusion occurred in case the patient presented one of the previously established criteria: 26 were diagnosed brain dead at admission and 50 had been transferred from another ICU, informed consent was not obtained from 149 patients and LOS in the ICU was less than 72 hours in 670 patients; 23 patients were excluded from analysis due to incomplete data collection for obtaining the NUTRIC score.

Patients that were excluded had similar age distribution than the recruited sample, but a significantly lower proportion were males (58.2\% vs $64.8 \% ; P=.002$ ) and had a significantly lower baseline APACHE II (median 17 vs $20 ; P=.009$ ) and SOFA (median 5 vs $7 ; P<.001$ ).

In the recruited sample, the median $\left(\mathrm{P}_{25}-\mathrm{P}_{75}\right)$ age at admission was 64 (51-75) years. Males predominated ( $\mathrm{n}=740,64.8 \% ; P<.001)$. The median $\left(\mathrm{P}_{25}-\mathrm{P}_{75}\right)$ baseline APACHE II and SOFA were 20 (14-26) and 7 (5-10), respectively. Primary admission diagnoses were respiratory $(\mathrm{n}=$ $262,23.0 \%$ ), sepsis $(n=230,20.2 \%)$ and trauma $(n=167,14.6 \%) ; 2$ or more co-morbidities were present in 393 (34.4\%) patients. Patients' baseline characteristics are summarized in Table 1.

The median $\left(\mathrm{P}_{25}-\mathrm{P}_{75}\right)$ of the days free of MV was 2 (1-4) days and LOS was 9 (5-15) days. At day 28, 243 of 1122 patients with known status were deceased (21.7\%; 95\% CI, 19.35-24.16).

The median $\left(\mathrm{P}_{25}-\mathrm{P}_{75}\right)$ of the NUTRIC score was 4 (3-6). There were 555 patients (48.6\%; 95\% CI, 45.67-51.45) at high nutritional risk (NUTRIC score $\geq 5$ ). Patients at high nutritional risk had higher median $\left(\mathrm{P}_{25}-\mathrm{P}_{75}\right)$ LOS 10 (5-16.5) days vs 8 (5-14) days $(P<.001)$, less median $\left(\mathrm{P}_{25}-\mathrm{P}_{75}\right)$ days free of MV $2(1-4)$ days vs $3(1-4)$ days $(P<.001)$, and higher 28 -day mortality $32.7 \%$ vs $11.2 \%(P<.001)$.

On logistic regression analysis, NUTRIC score $\geq 5$ was associated with longer $\operatorname{LOS}$ ( $\geq 9$ days) (OR 1.72; 95\% CI, 1.36-2.17; $P<.001 ; \mathrm{n}=1126$ ), less days free of MV ( $\leq 2$ days) (OR 1.46; 95\% CI, 1.16-1.85; $P=.002 ; \mathrm{n}=$ 1124) and higher 28-day mortality (OR 3.84; 95\% CI, 2.80-5.26; $P<$ $.001 ; \mathrm{n}=1122$ ). A NUTRIC score $\geq 5$ predicted 28-day mortality with AUC 0.658 (95\% CI, 0.620-0.696) (Supplementary Fig. S1), with a positive predictive value of $32.7 \%$ (95\% CI, 28.91-36.77) and a negative 
predictive value of $88.8 \%$ (95\% CI, 85.92-91.08) for 28-day mortality; its sensitivity was $73.25 \%$ (95\% CI, 67.36-78.42) and specificity 58.4\% (95\% $\mathrm{CI}, 55.07-61.58)$. The 28-day mortality prediction of the NUTRIC score, at its full range, calculated by a logistic regression model, is compared with the observed mortality rate in Fig. 1. The NUTRIC score as a full scale (0-9) predicted 28-day mortality with AUC 0.718 (95\% CI, 0.6850.752 ) (Fig. 2 and Table 2).

The comparison between the sample characteristics and the results obtained in this study with those of the original validation study are presented in Table 2.

\section{Discussion}

Most of the critically ill patients admitted to the ICU show malnutrition criteria [20]. Critical illness is typically associated with a catabolic stress state, skeletal muscle weakness, and as consequence with complications such as increased infectious morbidity, multiple-organ dysfunction, difficult weaning, prolonged hospitalization, and increased mortality. The nutrition therapy is thought to help to attenuate the metabolic response to stress, prevent oxidative cellular injury and modulate immune responses. Delivering early nutrition support, by the enteral route if possible, is a strategy that reduces disease severity, complications, decreases LOS in the ICU and improves patient outcomes [13]. It is known that a poor nutritional status is associated with a worse outcome, but the reverse may not be true since there are other factors associated with illness or with the status of the patient that can negatively influence the outcome regardless of nutritional status.

The NUTRIC score, firstly validated by Heyland et al, is the first nutritional risk assessment tool developed specifically for the ICU population that can identify patients at risk for malnutrition. Later Rahman et al validated the modified NUTRIC, which allows the exclusion of the IL-6 levels, if not available, to assess nutritional risk at admission.

This study was the first to characterize the nutritional risk of hospitalized patients in Portuguese polyvalent ICUs; the study considered a large sample that was representative of annual ICU admissions in

Table 1

Clinical characteristics of the sample of recruited patients. Values are median $\left(\mathrm{P}_{25}-\mathrm{P}_{75}\right)$ or $\mathrm{n}(\%)$.

\begin{tabular}{lc}
\hline & $\begin{array}{l}\text { Patients } \\
(\mathrm{n}=1143)\end{array}$ \\
\hline Age (years) & $64(51-75)$ \\
Gender & \\
Female & $402(35.2)$ \\
Male & $740(64.8)$ \\
Baseline APACHE II score & $20(14-26)$ \\
Baseline SOFA score & $7(5-10)$ \\
Days in hospital before ICU admission & $1(0-3)$ \\
BMI & $26.2(23.4-29.7)$ \\
Comorbidity & \\
Diabetes & $184(16.1)$ \\
Heart failure & $143(12.4)$ \\
Chronic pulmonary disease & $138(12.1)$ \\
Neoplasms & $152(13.3)$ \\
Chronic kidney disease & $69(6.0)$ \\
Brain stroke or transient ischemic event & $64(5.6)$ \\
Depression, anxiety or panic disorders & $55(4.8)$ \\
Hypertension & $47(4.1)$ \\
Chronic liver disease & $48(4.2)$ \\
Number of co-morbidities & $1(0-2)$ \\
Primary admission diagnosis & \\
Respiratory & $262(23.0)$ \\
Sepsis & $230(20.2)$ \\
Trauma & $167(14.6)$ \\
Post-operative conditions & $159(13.9)$ \\
Cardiovascular/vascular & $118(10.3)$ \\
Neurologic & $108(9.5)$ \\
Gastrointestinal & $49(4.3)$ \\
Metabolic & $36(3.2)$ \\
Renal & $12(1.1)$ \\
\hline
\end{tabular}

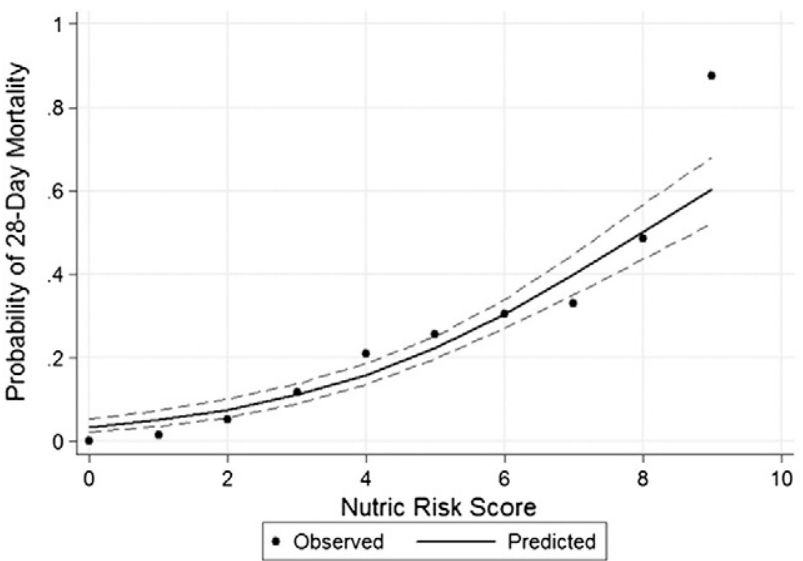

Fig. 1. The ability of the NUTRIC score to predict 28-day mortality. The graphic presents both the mortality rates which were observed (circles) and predicted by the logistic model (full line), the later with its 95\% confidence interval (broken line).

Portugal and it assessed prospectively the effectiveness of the modified NUTRIC score. High nutritional risk was present in half of the sample. As described in the original study $[14,18]$ in our work NUTRIC score was associated with the target clinical outcomes; it had low positive predictive value (32.7\%) and high negative predictive value (88.8\%) to predict 28 day mortality. In this study patients at high nutritional risk had higher LOS, fewer days free of MV and increased 28-day mortality. The mean NUTRIC score in our study (4.4) is slightly (but not significantly) lower than that of the original validation of the NUTRIC score (4.7) [14] though clearly lower than that of the second validation of the score (5.5) [18]. The participants in this Portuguese (national) sample show significant differences on the variables included in the NUTRIC score when compared to the original validation group [14]. In the present study, participants were significantly older, had higher proportions of lower APACHE II scores, higher SOFA scores and a significantly lower proportion of participants had two or more co-morbidities. These differences are due to both the clinical variability of the patients treated in the ICU and the large size of the recruited sample in the present study. Despite these differences, this study finds the same association already described between NUTRIC score and main clinical outcomes, which endorses the use of toll in clinical settings with different demographic characteristics from the original validation group.

It was more frequent for patients to be admitted to hospital for more than 1 day before ICU admission in this study, compared to the original validation study which could possibly be interpreted as a marker of compromised nutritional intake and worse nutritional status. The 28day mortality in this Portuguese national sample $(21.7 \%)$ is similar to that of the original validation of the NUTRIC score (23.1\%) [14] but it is lower than that observed in the second validation of the score (29\%) [18]. The AUC is significantly higher in the original validation study but in both studies it is classified as "adequate". In spite of the clinical and statistical differences found between this study and other studies $[14,18]$, the described performance of the NUTRIC score to predict 28day mortality is clinically similar across these studies and the values of the AUC fit into the category of "adequate". However, this study was not planned to reassess the validation of the instrument, rather to use it to characterize a sample of patients from a national sample of polyvalent ICUs.

The main strengths of this study are the large size of this national sample, the prospective evaluation of the patients using a standardized protocol and the clinical heterogeneity provided by the number of participant ICUs. The unblinded nature of the study may have affected the clinicians' intervention, but in the absence of a uniformized therapeutic protocol and with such a large number of patients, admitted to many ICUs all over the country, any influence of biased interventions is probably minimized. The absence of data of nutritional support (either 


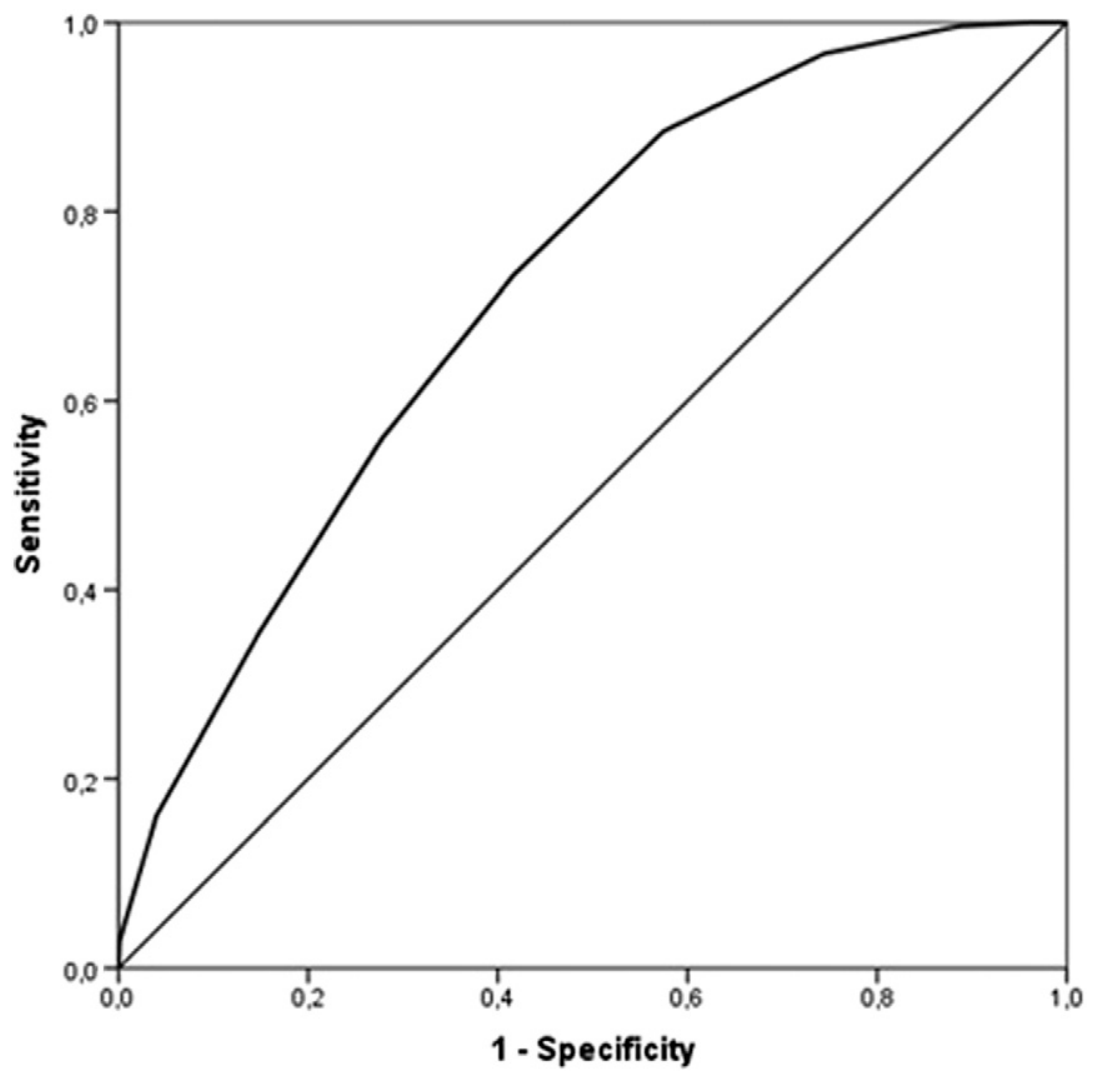

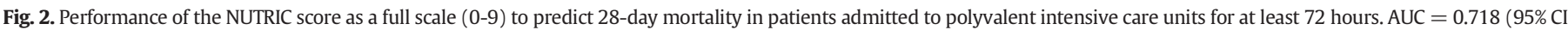
$0.685-0.752 ; \mathrm{n}=1122$ ).

enteral or parenteral) during ICU stay, the convenience sample and the potential heterogeneity of therapeutic approaches are other weaknesses. The use of this tool on a daily basis for critically ill patients seems to have the advantage of raising the importance of nutritional support, drawing attention to high-risk patients for malnutrition. Considering that patients with high nutritional risk are expected to have more difficulty in achieving their nutritional needs, especially protein needs, we believe protocols should be implemented to optimize delivery of nutritional therapy such as earlier introduction of motility agents higher threshold of tolerance to gastric residual volume, and a volume based strategy for delivery of enteral feeding (as opposed to an hourly based strategy). If the strategies mentioned fail to achieve the nutritional needs, those patients should be selected for supplementary parenteral nutritional. More prospective studies investigating witch nutritional interventions could positively modify the patients prognosis based on the NUTRIC score should be done in the near future.

Table 2

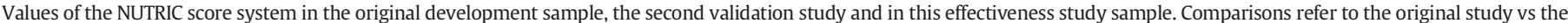
present effectiveness study.

\begin{tabular}{|c|c|c|c|c|c|c|}
\hline \multirow[t]{2}{*}{ Variables in NUTRIC score } & \multicolumn{2}{|c|}{ NUTRIC scoring system } & \multirow{2}{*}{$\begin{array}{l}\text { Original development } \\
\text { sample }(14) \\
(\mathrm{n}=598)\end{array}$} & \multirow{2}{*}{$\begin{array}{l}\text { Second validation } \\
\text { sample }(18) \\
(\mathrm{n}=1199)\end{array}$} & \multirow{2}{*}{$\begin{array}{l}\text { Effectiveness study } \\
\text { Sample } \\
(\mathrm{n}=1143)\end{array}$} & \multirow[b]{2}{*}{$P$} \\
\hline & Range & $\overline{\text { Points }}$ & & & & \\
\hline \multirow[t]{3}{*}{ Age } & $<50$ & 0 & $130(21.7)$ & 199 (16.6) & $255(22.3)$ & \\
\hline & $50-75$ & 1 & 345 (57.7) & $710(59.2)$ & $574(50.2)$ & .003 \\
\hline & $\geq 75$ & 2 & $123(20.6)$ & $290(24.2)$ & $314(27.5)$ & \\
\hline \multirow[t]{4}{*}{ APACHE II } & $<15$ & 0 & $111(18.6)$ & $48(4.0)$ & $292(25.6)$ & \\
\hline & $15-<20$ & 1 & 135 (22.6) & 157 (13.1) & $257(22.5)$ & \\
\hline & $20-<28$ & 2 & $226(37.8)$ & $508(42.4)$ & $374(32.7)$ & .007 \\
\hline & $\geq 28$ & 3 & $126(21.1)$ & $486(40.5)$ & $219(19.2)$ & \\
\hline \multirow[t]{3}{*}{ SOFA } & $<6$ & 0 & $220(36.8)$ & $157(13.1)$ & 376 (32.9) & \\
\hline & $6-<10$ & 1 & 247 (41.3) & $624(52.0)$ & $436(38.1)$ & .007 \\
\hline & $\geq 10$ & 2 & 131 (21.9) & 418 (34.9) & $331(29.0)$ & \\
\hline \multirow[t]{2}{*}{ Co-morbidities } & $0-1$ & 0 & $160(26.8)$ & $392(32.7)$ & 750 (65.6) & \\
\hline & $\geq 2$ & 1 & $438(73.2)$ & 807 (67.3) & $393(34.4)$ & $<.001$ \\
\hline \multirow[t]{2}{*}{ Days from hospital to ICU admission } & $<1$ & 0 & $375(62.7)$ & $757(63.1)$ & $466(40.6)$ & \\
\hline & $\geq 1$ & 1 & $223(37.3)$ & $442(36.9)$ & $678(59.4)$ & $<.001$ \\
\hline \multirow[t]{2}{*}{ IL6 } & $<400$ & 0 & $489(81.8)$ & - & - & \\
\hline & $\geq 400$ & 1 & $109(18.2)$ & - & - & \\
\hline Score range (IQR) & & & $0-10(3-6)$ & $0-9(4-7)$ & $0-9(3-6)$ & \\
\hline Score mean (SD) & & & $4.7(2.2)$ & $5.5(1.6)$ & $4.4(2.1)$ & .086 \\
\hline AUC & & & 0.783 & 0.783 & 0.718 & .232 \\
\hline Gen R-Squared & & & 0.169 & 0.169 & 0.103 & \\
\hline Gen Max-rescaled R-Squared & & & 0.256 & 0.256 & 0.158 & \\
\hline
\end{tabular}




\section{Conclusion}

The modified NUTRIC score, the first nutritional risk assessment tool developed and validated specifically for critically ill patients, demonstrated that in ICU Portuguese patients, despite presenting different characteristics from the original validated sample, a good correlation with main clinical outcomes.

The modified NUTRIC score can be used widely and systematically, contributing to discriminate ICU patients at high nutritional risk. The cross-cultural adaptation of NUTRIC score demonstrated translation reliability and is acceptable to be used in critically ill patients.

Supplementary data to this article can be found online at http://dx. doi.org/10.1016/j.jcrc.2016.08.001.

\section{Statement of authorship}

All authors were responsible for the study design, writing and revising of the manuscript. MA and DA were also responsible for the statistical analysis and data interpretation.

\section{Conflict of interest statement}

None declared.

\section{Funding sources}

This research did not receive any specific grant from funding agencies in the public, commercial, or not-for-profit sectors.

\section{Acknowledgements}

The authors acknowledge the collaboration of the ICUs that participated in this multicenter national study-.

Portuguese NUTRIC Study Group:

Centro Hospitalar de Lisboa Central (CHLC) H. de São José.

- Unidade de Urgência Médica (UUM) - Coordinator: Bento L. MD; Local investigators: Francisco A.R. MD; Graça A. MD.

- UCI Polivalente 1 (UCIP 1) Coordinator: Lopes V. MD; Local investigators: Simas A. MD; Miranda I. MD; Vera Cruz M. MD; Shumanska Z. MD.

Centro Hospitalar de Lisboa Norte (CHLN) H Santa Maria.

- Unidade de Isolamento Respiratório/Doenças Infecciosas (UIR/ UCIDI) Coordinator: Neves J. MD; Local investigator: Policarpo S. RD.

- UCI Respiratórios (UCIR) Coordinator: Monteiro J. MD; Local investigator: Martins T. RD.

Centro Hospitalar de São João (CHSJ) H de São João.

- UCI Polivalente da Urgência Coordinator: Paiva A. MD, PhD; Local investigators: Gomes J.P. MD; Trindade L. RN.

- UCI Polivalente Coordinator: Oliveira T. MD; Local investigator: Aguiar L. MD.

Instituto Português de Oncologia do Porto Francisco Gentil.

- Serviço de Cuidados Intensivos Coordinator: Faria F. MD; Local investigator: Cabral S. RN.

Centro Hospitalar de Entre o Douro e Vouga - H. de São Sebastião Santa Maria da Feira.

- UCI Polivalente Coordinator: Amaro P. MD; Local investigator: Rodrigues P.R. MD.

Centro Hospitalar de Vila Real/Peso da Régua -H. de São Pedro de Vila Real.

- UCI Polivalente Coordinator: Esteves F.S. MD; Local investigators: Santos A. MD; Gonçalves L. MD

Centro Hospitalar Universitário de Coimbra.

- UCI Coordinator: Pimentel J. MD, PhD; Local investigator: Martins P. MD PhD.
Centro Hospitalar Tondela-Viseu - H. de São Teotónio.

- UCI Coordinator: Saraiva J.P. MD; Local investigator: Melo E. MD. Unidade Local de Saúde do Baixo Alentejo.

- UCI Polivalente Coordinator: Vaz J. MD; Local investigators: Barahona I. MD; Carvalho A. MD.

Hospital do Litoral Alentejano - Santiago do Cacém.

- UCI Coordinator: Moreira P. MD; Local investigator: Vilas M.J. MD. Centro Hospitalar do Algarve - H. de Faro.

- Serviço de Medicina 1. Departamento de Emergência, Urgência e Cuidados Intensivos Coordinator: Granja C. MD, PhD; Local investigator: Sousa A. MD. o Santo - Ponta Delgada, Açores.

- UCI Coordinator: Maia D.F. MD; Local investigators: Henriques M. MD; Carvalho R. RN.

\section{References}

[1] White JV, Guenter P, Jensen G, Malone A, Schofield M, Academy Malnutrition Work $G$, et al. Consensus statement: academy of nutrition and dietetics and American Society for Parenteral and Enteral Nutrition: characteristics recommended for the identification and documentation of adult malnutrition (undernutrition). JPEN J Parenter Enteral Nutr 2012;36(3):275-83.

[2] Edington J, Boorman J, Durrant ER, Perkins A, Giffin CV, James R, et al. Prevalence of malnutrition on admission to four hospitals in England. The malnutrition prevalence group. Clin Nutr 2000;19(3):191-5.

[3] Correia MI, Waitzberg DL. The impact of malnutrition on morbidity, mortality, length of hospital stay and costs evaluated through a multivariate model analysis. Clin Nutr 2003;22(3):235-9.

[4] Schneider SM, Veyres P, Pivot X, Soummer AM, Jambou P, Filippi J, et al. Malnutrition is an independent factor associated with nosocomial infections. Br J Nutr 2004; 92(1):105-11.

[5] Matos L, Teixeira MA, Henriques A, Tavares MM, Alvares L, Antunes A, et al. Nutritional status recording in hospitalized patient notes. Acta Med Port 2007;20(6): 503-10.

[6] Sorensen J, Kondrup J, Prokopowicz J, Schiesser M, Krahenbuhl L, Meier R, et al. EuroOOPS: an international, multicentre study to implement nutritional risk screening and evaluate clinical outcome. Clin Nutr 2008:27(3):340-9.

[7] Detsky AS, McLaughlin JR, Baker JP, Johnston N, Whittaker S, Mendelson RA, et al. What is subjective global assessment of nutritional status? 1987. Classical article. Nutr Hosp 2008;23(4):400-7.

[8] Malnutrition Advisory G. A consistent and reliable tool for malnutrition screening. Nurs Times 2003;99(46):26-7.

[9] Kruizenga HM, Seidell JC, de Vet HC, Wierdsma NJ, van Bokhorst-de van der Schueren MA. Development and validation of a hospital screening tool for malnutrition: the short nutritional assessment questionnaire (SNAQ). Clin Nutr 2005;24(1): 75-82.

[10] Ferguson M, Capra S, Bauer J, Banks M. Development of a valid and reliable malnutrition screening tool for adult acute hospital patients. Nutrition 1999;15(6): 458-64.

[11] Anthony PS. Nutrition screening tools for hospitalized patients. Nutr Clin Pract 2008; 23(4):373-82.

[12] Kondrup J, Rasmussen HH, Hamberg O, Stanga Z, Ad Hoc EWG. Nutritional risk screening (NRS 2002): a new method based on an analysis of controlled clinical trials. Clin Nutr 2003;22(3):321-36.

[13] Preiser JC, van Zanten AR, Berger MM, Biolo G, Casaer MP, Doig GS, et al. Metabolic and nutritional support of critically ill patients: consensus and controversies. Crit Care 2015;19:35.

[14] Heyland DK, Dhaliwal R, Jiang X, Day AG. Identifying critically ill patients who benefit the most from nutrition therapy: the development and initial validation of a novel risk assessment tool. Crit Care 2011;15(6):R268.

[15] Heyland DK. Critical care nutrition support research: lessons learned from recent trials. Curr Opin Clin Nutr Metab Care 2013;16(2):176-81.

[16] Knaus WA, Draper EA, Wagner DP, Zimmerman JE. APACHE II: a severity of disease classification system. Crit Care Med 1985;13(10):818-29.

[17] Moreno R, Vincent JL, Matos R, Mendonca A, Cantraine F, Thijs L, et al. The use of maximum SOFA score to quantify organ dysfunction/failure in intensive care. Results of a prospective, multicentre study. Working group on sepsis related problems of the ESICM. Intensive Care Med 1999;25(7):686-96.

[18] Rahman A, Hasan RM, Agarwala R, Martin C, Day AG, Heyland DK. Identifying critically-ill patients who will benefit most from nutritional therapy: further validation of the "modified NUTRIC" nutritional risk assessment tool. Clin Nutr 2016; 35(1):158-62.

[19] Beaton DE, Bombardier C, Guillemin F, Ferraz MB. Guidelines for the process of cross-cultural adaptation of self-report measures. Spine 2000;25(24): 3186-91.

[20] Coltman A, Peterson S, Roehl K, Roosevelt H, Sowa D. Use of 3 tools to assess nutrition risk in the intensive care unit. JPEN J Parenter Enteral Nutr 2015;39(1): 28-33. 